Gut, 1971, 12, 110-118

\title{
The value of electron microscope studies in diagnosing malignant change in ulcerative colitis
}

\author{
JANET A. JENKINSON AND I. M. P. DAWSON \\ From the Udall Street Laboratories, Westminster Hospital Medical School and Hospital, London
}

SUMMARY Ultrastructural and histological appearances were studied in normal rectal and colonic mucosa, in the mucosa immediately adjacent to neoplasms (transitional mucosa), in neoplasms themselves, and in ulcerative colitis to try to determine whether any changes found in colitis might be recognized as premalignant.

Two of the ulcerative colitis specimens showed histological evidence of premalignant change and these changes were reflected at the ultrastructural level. No electron microscopical premalignant changes were found which could not more easily be detected by simple histology, and it is concluded that attempts to detect premalignant changes in ulcerative colitis using electron microscopical techniques are likely to be unrewarding.

Many adenocarcinomas of the colon and rectum appear to develop from previously normal epithelium, and sometimes characteristic histological alterations from normal in the epithelium herald the change. Ulcerative colitis is the only common inflammatory condition in which a high incidence of malignant change is recorded especially in those patients who have had inflammatory mucosal changes for more than 10 years (Dawson and Pryse-Davies, 1959; Hinton, 1966). This suggests that prolonged mucosal inflammation or repeated regeneration may lead to epithelial changes which either initiate malignant change or predispose to it. In rectal biopsies from patients with ulcerative colitis Morson and Pang (1967) have described precancerous changes visible under the light microscope which include irregularity in cell size and shape, nuclear hyperchromatism with prominent nucleoli, and diminution in mucus secretion. These changes are, however, patchy and variable, and a single biopsy may fail to show them.

It has been shown histochemically in our laboratories that epithelial mucosubstances show variation from normal in distribution and chemical structure, particularly with regard to sulphation, both in ulcerative colitis (Filipe and Dawson, 1970) and at the edge of carcinomas in normal bowel (Filipe, 1969), and it seems reasonable to suggest that ultrastructural changes might be present which would parallel these observations. If changes occurred in Received for publication 6 Ocrober 1970. the apparently normal epithelium immediately adjacent to a carcinoma which might be interpreted as 'characteristic' ultrastructural premalignant change, then changes found in colitic epithelium could be judged as premalignant if they showed a similar pattern. We have therefore studied material from carcinomas of colon and rectum to discover the range of appearances in normal epithelium, and from the edge of carcinomas and from patients with ulcerative colitis in an attempt to recognize precancerous changes in the former and so diagnose them in the latter.

\section{Materials and Methods}

Specimens of colonic and rectal mucosa were obtained from 11 patients operated on for carcinoma of the colon or rectum and from seven patients undergoing surgery for clinical ulcerative colitis. In the former cases specimens were taken from three areas: the carcinoma, the region immediately adjacent to the carcinoma (the 'transitional' zone), and a region more than $10 \mathrm{~cm}$ from the carcinoma, which was considered 'normal' and used as control material. Blocks from these zones were fixed in $10 \%$ formalin, embedded in paraffin wax, and sectioned at $6 \mu$ for routine histology. Small pieces of mucosa (not bigger than $5 \mathrm{~mm} \times 5 \mathrm{~mm}$ ) were fixed for three to four hours at $4^{\circ} \mathrm{C}$ in $3 \%$ glutaral-

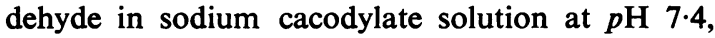
washed overnight in sucrose-cacodylate solution, 
postfixed in Millonigs buffered osmium tetroxide, and dehydrated in an ascending ethanol series, being maintained at $4^{\circ} \mathrm{C}$ until the absoute ethanol stage. The specimens were then cleared in propylene oxide and embedded in Araldite.

Thick (150-250 $\mathrm{m} \mu$ ) and thin (up to $90 \mathrm{~m} \mu$ ) transverse sections across the middle regions of the crypts were cut on a Sorvall Porter Blum MT-1 ultramicrotome, the thick sections being stained with borax-toluidine blue for light microscopy. Thin sections were stained with uranyl acetate and lead citrate and were viewed in a Zeiss EM9 electron microscope. Electron micrographs were taken on Ilford line N4E50 film at magnifications up to 40,000 .

In the seven colitics, longitudinal blocks were taken from defined sites for routine histology (Dawson and Pryse-Davies, 1959). From one to

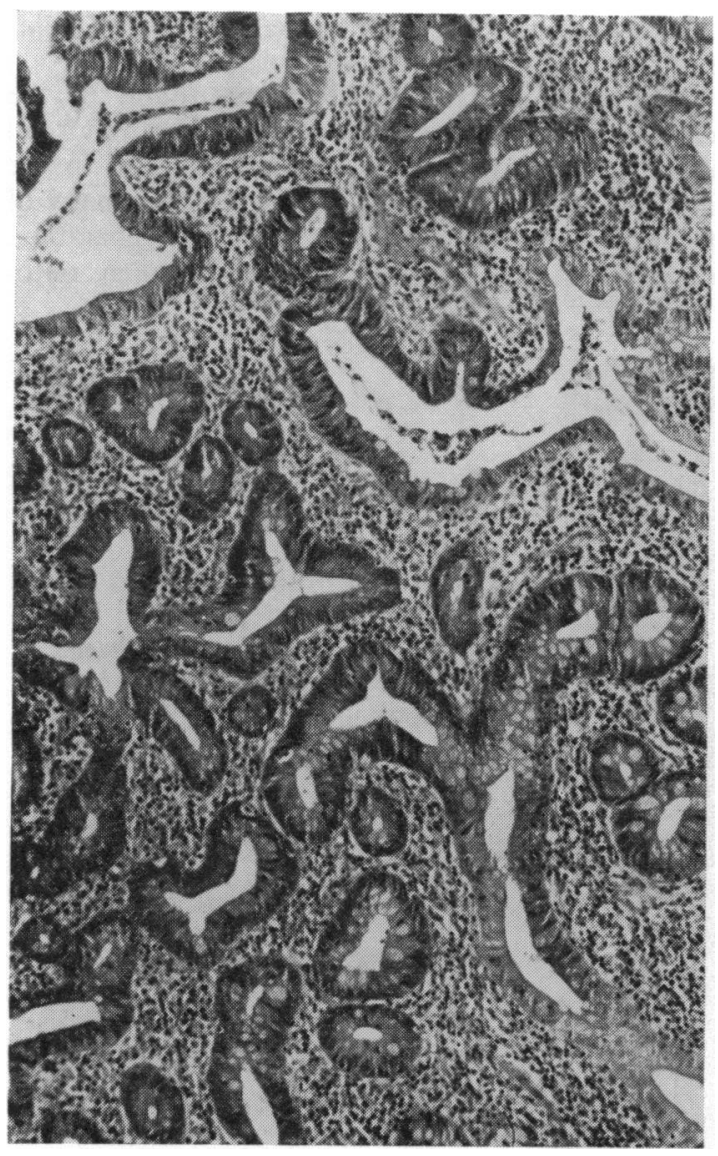

Fig. 1, Ulcerative colitis with polypoidal pattern of precancerous change. Haematoxylin and eosin, $\times 110$. three random samples of mucosa from affected zones adjacent to one or more of the blocks were taken for electron microscopy and processed as already described. Additional normal mucosa from other patients who underwent resection of the large bowel for carcinoma was used for controls.

A total of 60 specimens were examined, of which 26 were normal mucosa, 11 were carcinoma, 11 were transitional mucosa, and 12 were mucosa from ulcerative colitis cases of varying degrees of severity. Fifteen of these specimens were taken from the rectum and 45 from various regions of the colon.

\section{Observations}

HISTOLOGY

Two of the patients diagnosed clinically as suffering

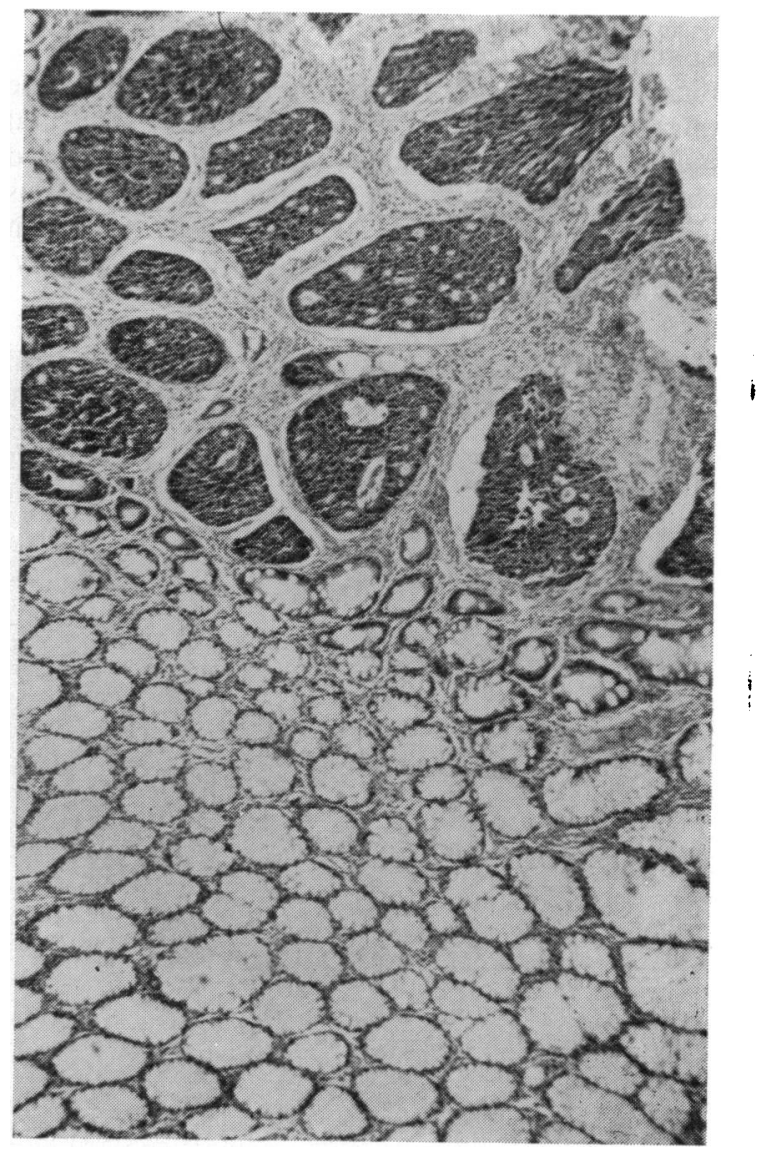

Fig. 2 Undifferentiated carcinoma of the rectum. Note the abrupt change from normal to neoplastic epithelium. $H \& E, \times 110$. 
from ulcerative colitis were found on histological examination to have Crohn's disease and were rejected from the survey. The remaining five showed the characteristic histological changes of chronic active ulcerative colitis, with evidence of concurrent ulceration and epithelial regeneration. Two of them, one of 10 and one of eight years' standing, showed the polypoidal pattern of precancerous change (Fig. 1) described by Dawson and Pryse-Davies (1959) and Morson and Pang (1967). None showed precancerous changes in flat mucosa (Morson and Pang, 1967).

Histological studies on the transitional mucosa immediately adjacent to the 11 carcinomas showed no recognizable abnormality. The carcinomas appeared to arise abruptly from the epithelium (Fig. 2) and the immediately adjacent low-power field exactly resembled the epithelium from supposed normal mucosa taken at $10 \mathrm{~cm}$ or more from the carcinoma.

\section{ELECTRON MICROSCOPY}

Since no significant differences were found in the ultrastructure of the normal colonic and rectal mucosa these two regions have been considered collectively. Our studies confirmed those of previous authors (Florey, 1960; Hampton, 1960; Shearman and Muir, 1960; Bierring, 1962; Fisher and Sharkey,
1962; Freeman, 1962; Birbeck and Dukes, 1963; Pittman and Pittman, 1966) and our findings in the carcinoma specimens were also comparable with previous descriptions (Fisher and Sharkey, 1962; Birbeck and Dukes, 1963). We therefore propose to describe only those normal features which are altered in carcinoma or ulcerative colitis.

In the normal mucosa the goblet and principal cells are columnar and contain roughly round or ovoid nuclei which may have one or two shallow indentations in their surface. The cells usually appear to have been normally active at the time of fixation and evidence of degeneration is only occasionally found. The relationship of adjacent cells to each other is characteristic. Towards the apices the epithelial cells are fairly straight-sided and are in very close contact with each other, terminal bars being clearly visible. At the lower lateral borders, however, the cells have many slender processes which interdigitate with those of adjacent cells, and often quite wide intercellular spaces may be left between them (Fig. 3).

The main ultrastructural changes found in the carcinoma specimens are evidence of abnormal mucin production (Fig. 4) and a decrease in the number of goblet cells with evidence that many of both these and the principal cells are degenerating. (The criteria of degeneration are swollen mito-

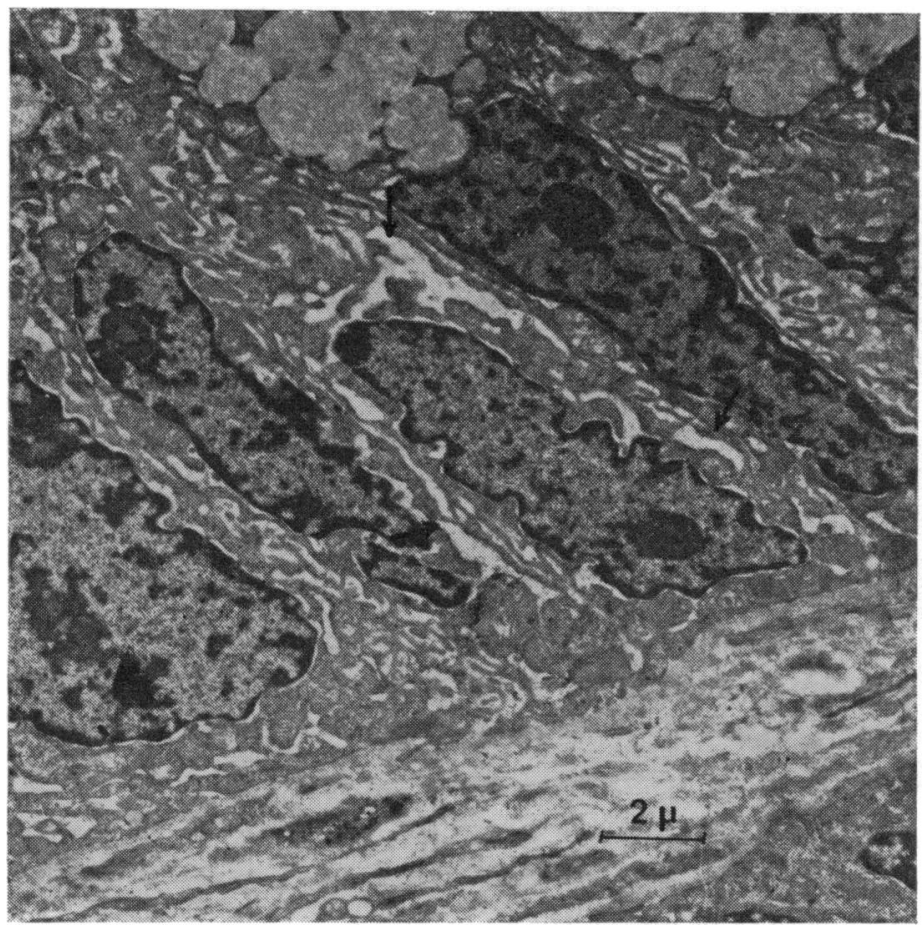

Fig. 3 Normal colon, showing the intercellular spaces between the bases of the cells (at arrows). 
chondria, disrupted Golgi apparatus and endoplasmic reticulum and the presence of autolysosomes and many small empty vesicles.) There is often also a marked change in the degree of contact between adjacent cells. The terminal bars are frequently still visible apically, although the space between the two cell borders is enlarged. Towards the bases of the cells, however, the reverse occurs and the intercellular spaces tend to diminish in size (Fig. 5).

Electron microscopical studies on the transitional mucosa adjacent to the carcinomas confirm the impression gained by light microscopy of an abrupt change from normal epithelium to carcinoma. No differences in fine structure between this region and the normal epithelium can be found except for the occasional appearance of irregularly shaped nuclei.

The changes in the ulcerative colitis cases vary not only from one specimen to the next, but also within a single specimen. Quite often the appearances of cells in two adjacent crypts is quite different. The two patients whose epithelium showed possible precancerous changes at the light microscope level also showed extensive changes ultrastructurally. In both cases, however, many apparently normal crypts were also seen. The changes that were found in these two cases were stratified, enlarged, and irregularly shaped nuclei (visible by both light and electron microscopy) and changes in the relationships of adjacent cells. In the abnormal crypts the apices

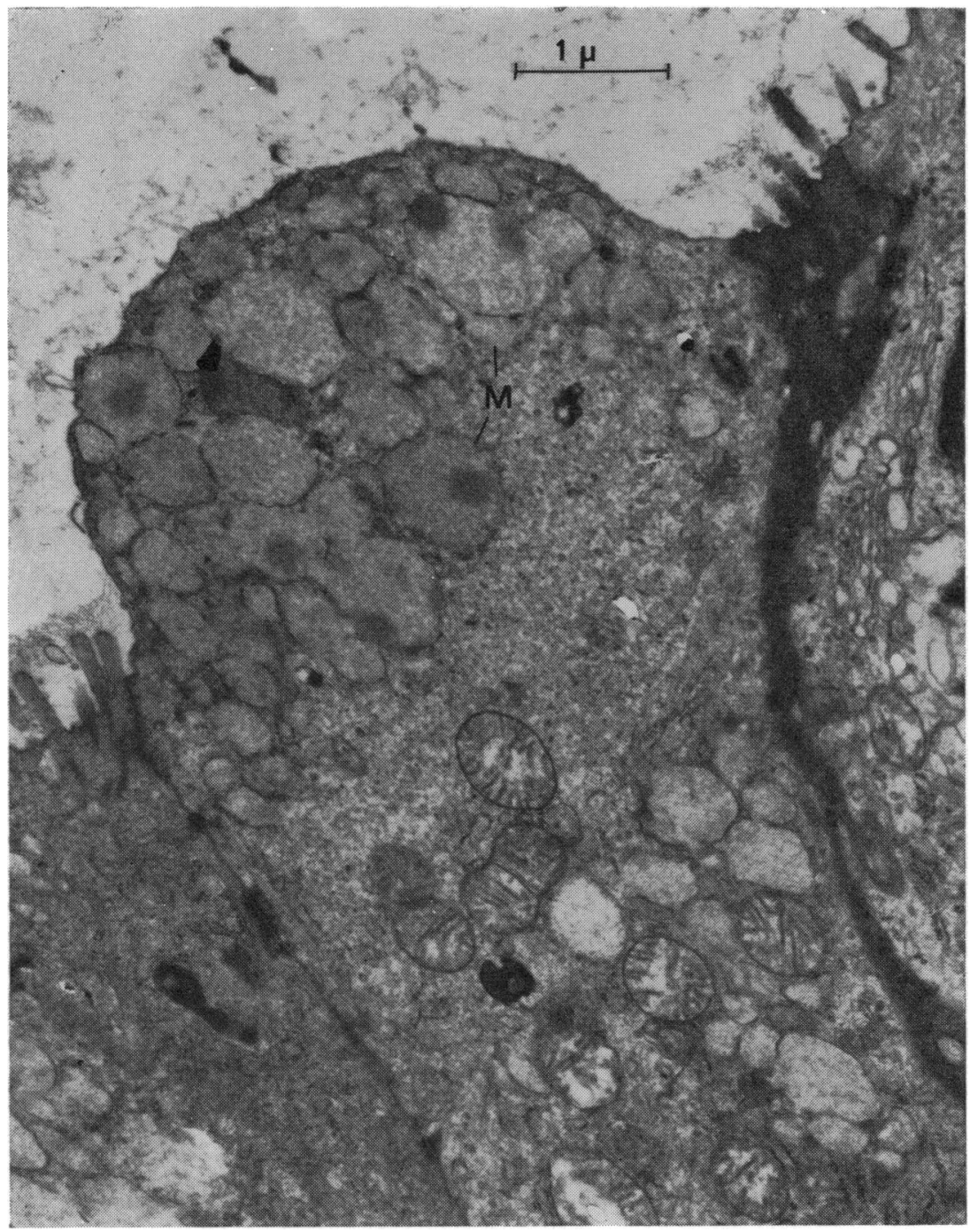

Fig. 4 Carcinoma of the colon. Part of a goblet cell with abnormal goblet formation (M = mucin). 
of adjacent cells are still in close contact, while the intercellular spaces at the bases of the cells may become so distended that the cells are forced apart (Fig. 6). It was also noted that in these two cases a larger than normal proportion of apparently degenerating goblet and principal cells is present and frequently cells show signs of erosion at the luminal surface (Fig. 7).

The other three cases studied revealed no precancerous changes by light microscopy, but electron

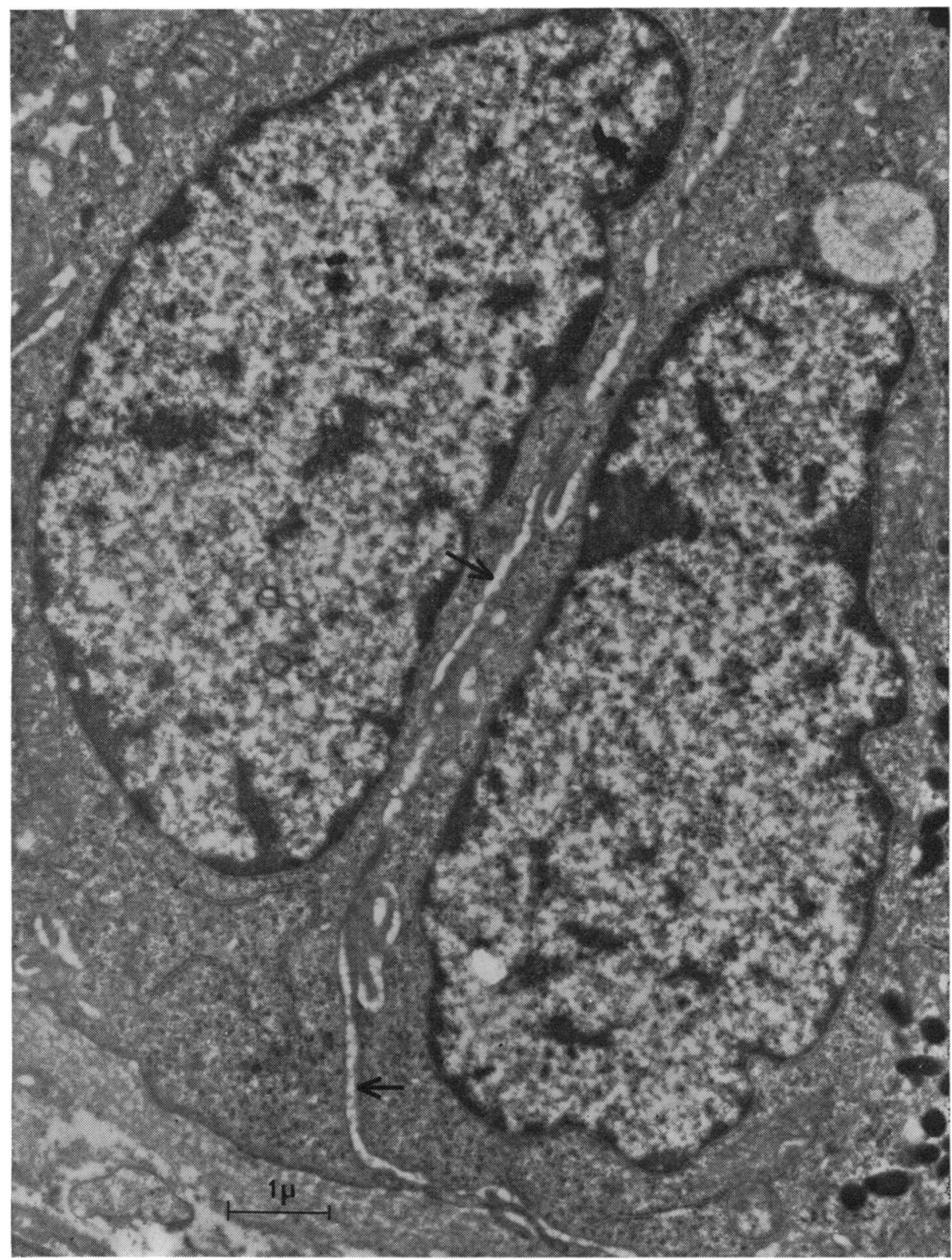

Fig. 5 Carcinoma of the colon. Bases of epithelial cells showing that intercellular spaces have diminished (at arrows). Compare with Figure 3. 
microscopical examination of one of them (with ulcerative colitis of 10 years' standing) suggests that this may be a false impression. Three separate specimens were taken from the resected bowel of this patient for electron microscopy. Two of them show apparently normal cell structure in the epithelium. The third has many apparently normal crypts, but several show stratification and irregular shaping of the nuclei. In these crypts the intercellular spaces are enlarged, many cells appear to

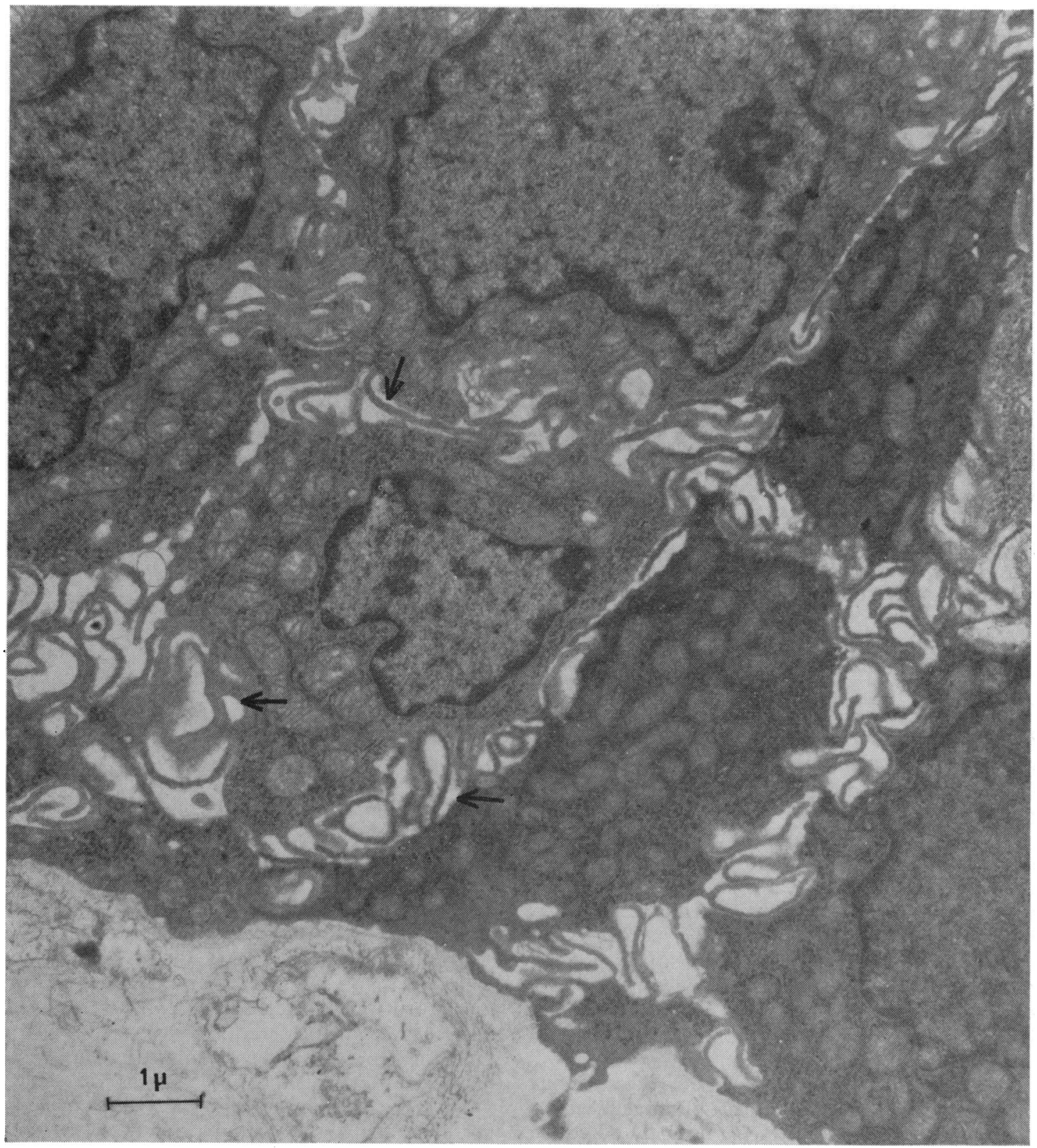

Fig. 6 Ulcerative colitis. Basal portions of epithelial cells showing distended intercellular spaces (at arrows). Compare with Figure 3. 


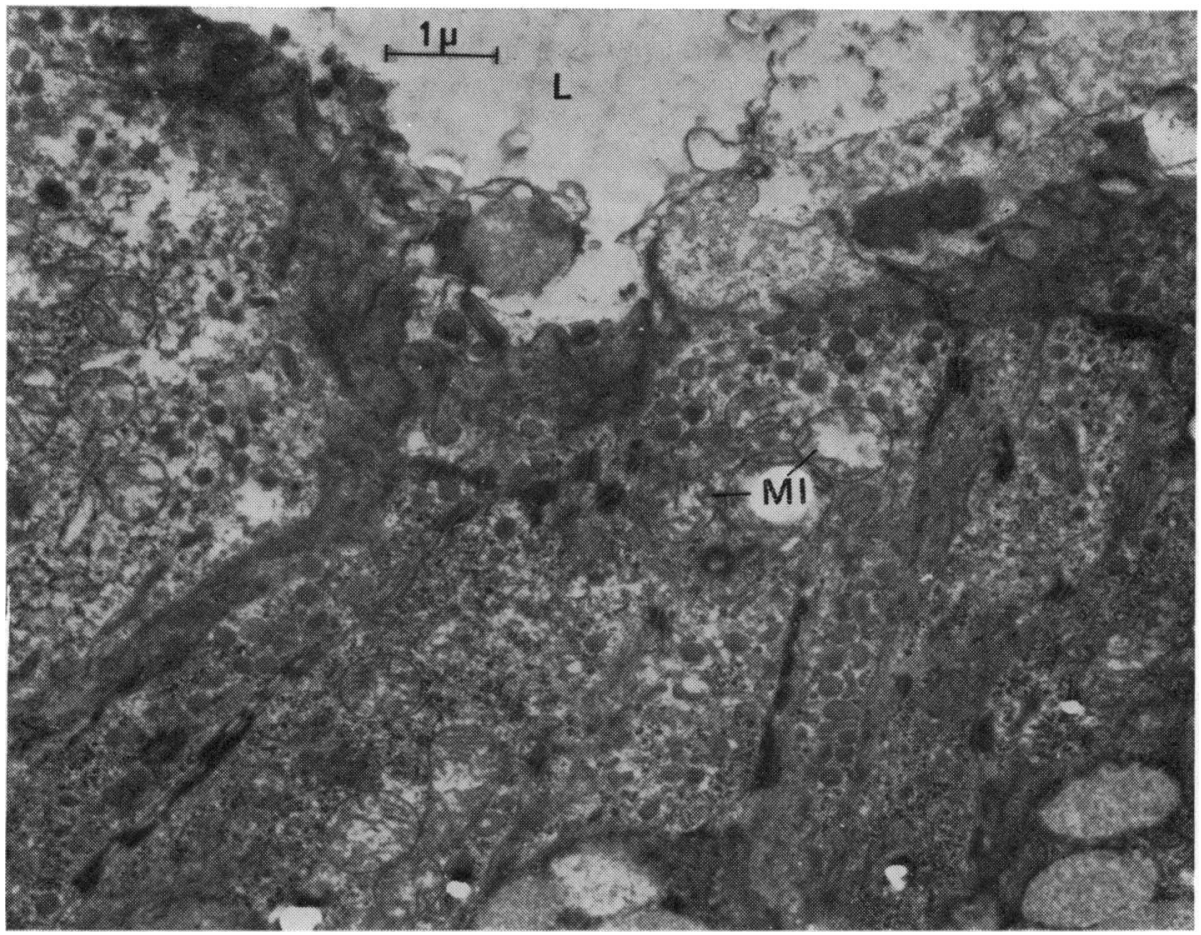

Fig. 7 Ulcerative colitis. Apical portions of epithelial cells showing swollen mitochondria (MI) and erosion of the luminal surface $(\mathrm{L}=$ lumen $)$.

be degenerating and the luminal surfaces of the cells are frequently eroded. This suggests a precancerous state which was not detected by light microscopy.

The remaining two cases (one of five years' and one of two months' standing) appear fairly normal ultrastructurally. No stratification of the cells can be seen and most of the nuclei appear normal in size and shape (one or two in one specimen were slightly irregular, but not markedly so). In both of these cases there appears to be a smaller proportion of degenerating cells (although there are more than in normal epithelium), but the intercellular spaces are slightly enlarged.

In an attempt to detect other possible precancerous changes a careful count was made of the numbers of principal and goblet cells in the normal and colitic specimens to see if there was any change in their proportions. This reveals that there is no significant difference in the proportions of these two cell types in ulcerative colitis.

\section{Discussion}

The work described here is an attempt to answer two questions which are related. First, are the histological changes of precancer in ulcerative colitis which Morson and Pang (1967) described reflected in changes in the electron microscopic appearances and, if so, could ultrastructural changes perhaps be detected before histological ones? Second, leaving aside the criteria of Morson and Pang, are there ultramicroscopic changes in the transitional mucosa adjacent to carcinomas which might be regarded as precancerous, and, if found in biopsies from patients with colitis, might give an indication of malignant potential?

We were unusually fortunate in that two of the first seven patients we studied showed the precancerous changes described by Morson and Pang (1967) when viewed with light microscopy. The other three colitics and both cases of Crohn's disease showed no histological evidence of precancer. 
Our electron microscopic observations on the two colitics showing histological precancerous changes confirmed the light microscopy evidence (enlarged, irregularly shaped nuclei and cell stratification) but revealed little beyond this except the degeneration of a larger than normal proportion of cells and the enlargement of intercellular spaces. Other workers have also found that degeneration of cells may occur. Morson and Pang (1967) and Filipe (1969) have reported a decrease in mucin production due either to a decrease in the number of goblet cells or to malfunctioning on their part. Greco, Lauro, Fabbrini, and Torsoli (1967) have also suggested an ultimate decrease in the number of goblet cells in advanced ulcerative colitis and suggest that this may be a premalignant change. Our studies appear to agree more with those of Gonzalez-Licea (1966) and Gonzalez-Licea and Yardley (1966) in that it is not the goblet cells alone that are degenerating. We have found an equal number of degenerating principal cells and no change in the overall proportions of the cells. The fact that more cells are degenerating than in the normal epithelium may indicate precancer, but since we found signs of this in all our patients, including one with a very short history of colitis, we feel that it is probably not indicative of precancerous change. The fact that this is not a constant feature in every crypt would also make it an unreliable criterion of premalignancy in biopsy material.

The ballooning of the intercellular spaces has also been noted by other authors (Gonzalez-Licea, 1966; Gonzalez-Licea and Yardley, 1966) who state that it is evidence of intercellular oedema and is probably an inflammatory reaction rather than a premalignant change. Our own observations show that although this causes the cells to separate, it is almost certainly a different process than that causing cell separation in carcinomas. In the latter the intercellular spaces tend to be smaller rather than larger and it is the apices of the cells which separate. If this is the case then enlargement of the intercellular spaces cannot be regarded as evidence of precancer.

Therefore it appears from our investigations that there are no premalignant changes in ulcerative colitis which cannot also be detected by simple histology. Although one of our specimens showed apparently premalignant changes which had not been detected by light microscopy we do not feel that this is evidence that it is possible to detect the changes earlier by electron microscopy, but rather a reflection on the element of chance involved in detecting these changes. In all the specimens we studied we found many apparently unaffected crypts, indicating that precancerous changes occur only at scattered foci in the affected area and are 2 not overall changes. Thus a single biopsy would not necessarily show any changes that had occurred. Our study, however, was confined to the epithelium and it is possible that overall changes may occur in the submucosa which could denote a malignant potential. Donnellan (1966), Gonzalez-Licea (1966), and Gonzalez-Licea and Yardley (1966) have made some preliminary studies in this field.

The finding that there were no visible histological variations from normal in the transitional epithelium adjacent to a carcinoma is in accord with general experience, though perhaps surprising in view of the fact that histochemical studies (Filipe, 1969; Filipe and Dawson, 1970) have shown that there are considerable alterations in sulphated and non-sulphated mucosubstances in this zone. The absence of significant ultrastructural changes in the transitional mucosa, however, seems to indicate that there is no morphological difference between sulphated, non-sulphated, and neutral mucosubstances and that alterations in the proportions in which they are produced (Filipe, 1969) are not reflected in structural cytological changes.

From these studies we conclude that attempts at the detection of incipient malignant change in ulcerative colitis using electron microscopic techniques on biopsy material is likely to be unrewarding.

The authors would like to thank the surgical staff of the Gordon Hospital (Westminster Group), and especially Mr S. O. Aylett for access to surgical material, and Miss Jill Ashby for secretarial assistance. The whole study was supported by a grant from the Cancer Research Campaign which is gratefully acknowledged.

\section{References}

Bierring, F. (1962). Electron microscopic observations on the mucus production in human and rat intestinal goblet cells. Acta path microbiol. scand., 54, 241-252.

Birbeck, M. S. C., and Dukes, C. E. (1963). Electron microscopy of rectal neoplasms. Proc. roy. Soc. Med., 56, 793-797.

Dawson, I. M. P., and Pryse-Davies, J. (1959). The development of carcinoma of the large intestine in ulcerative colitis. Brit. $J$. Surg., 47, 113-128.

Donnellan, W. L. (1966). Early histological changes in ulcerative colitis. A light and electron microscopic study. Gasteroenterology, 50 , 519-540.

Filipe, M. I. (1969). Value of histochemical reactions for mucosubstances in the diagnosis of certain pathological conditions of the colon and rectum. Gut, 10, 577-586.

Filipe, M. I., and Dawson, I. (1970). The diagnostic value of mucosubstances in rectal biopsies from patients with ulcerative colitis and Crohn's disease. Gut, 11, 229-234.

Fisher, E. R., and Sharkey, D. A. (1962). The ultrastructure of colonic polyps and cancer with special reference to the epithelial inclusion bodies of Leuchtenberger. Cancer (Philad.), 15, 160170.

Florey, H. W. (1960). Electron microscopic observations on goblet cells of the rat's colon. Quart. J. exp. Physiol, 45, 329-336.

Freeman, J. A. (1962). Fine structure of the goblet cell mucous secretory process. Anat. Rec., 144, 341-345. 
Gonzalez-Licea, A. (1966). Ulcerative colitis: eleztron microscopic observations on rectal biopsy. Dis. Colon Rect., 9, 417-419.

Gonzalez-Licea, A., and Yardley, J. H. (1966). Nature of the tissue reaction in ulcerative colitis. Light and electron microscopic findings. Gastroenterology, 51, 825-840.

Greco, V., Lauro, G., Fabbrini, A., and Torsoli, A. (1967). Histochemistry of the colonic epithelial mucins in normal subjects and in patients with ulcerative colitis. Gut, 8, 491-496.

Hampton, J. C. (1960). An electron microszopic study of mouse colon. Dis. Colon Rect., 3, 423-440.
Hinton, J. M. (1966). Risk of malignant change in ulcerative colitis. Gut, 7, 427-432.

Morson, B. C., and Pang, L. S. C. (1967). Rectal biopsy as an aid to cancer control in ulcerative colitis. Gut, 8, 423-434.

Pittman, F. E., and Pittman, J. C. (1966). An electron microscopic study of the epithelium of normal human sigmoid colonic mucosa. Gut, 7, 644-661.

Shearman, D. J. C., and Muir, A. R. (1960). Observations on the secretory cycle of goblet cells. Quart. J. exp. Physiol., 45, 337-342. 